\title{
Le contrôle de la fiscalité des ressources naturelles et minières du Togo
}

\author{
Pr. Akodah Ayewouadan
}

Le « Kevazingo Gate » au Gabon et le scandale relatif aux hydrocarbures au Sénégal ${ }^{1}$ ont jeté une lumière crue sur les relations tourmentées que les pays africains entretiennent avec leurs ressources naturelles et minières. L'une des illustrations de ces relations tourmentées réside dans le délicat contrôle de la fiscalité des ressources naturelles et minières.

L'importance de la mobilisation de recettes fiscales internes pour les pays en développement n'est plus à démontrer. L'une des sources les plus importantes de ces recettes reste le secteur des ressources naturelles et le secteur minier. Sur le secteur minier par exemple, plus de la moitié des pays africains sont producteurs de ressources minérales. Sur cinquante-quatre (54) pays, vingt (20) sont dits riches en ressources naturelles d'après les critères du FMI ${ }^{2}$. Toutefois, ces pays restent pauvres selon la grille de lecture onusienne, ce qui pose le débat de la capacité des gouvernements africains à capter la rente apportée par les ressources naturelles et minières. Il en va de même pour la méthodologie utilisée pour cette captation. Ce débat qui, dans le cadre de cette étude est restreint au Togo, pourrait légitimement être élargi à d'autres pays africains.

Les ressources naturelles ont souvent figuré des aimants à problèmes. D'aucuns n'ont pas manqué de parler de la «malédiction des ressources naturelles » ${ }^{3}$. Cette malédiction frapperait, selon cette théorie, les États richement dotés par la nature. Au vrai, les institutions étatiques ont souvent œuvré, avec plus ou moins de réussite, pour transformer cette malédiction en bénédiction. Ils ont pour la plupart institué le système fiscal et sa mise en œuvre comme une arme de choix pour lutter contre ce mal congénital. La fiscalité des ressources naturelles et minières au Togo est un enchevêtrement complexe de prélèvements, aux propriétés très variées. Elle trouve sa source dans le code minier ${ }^{4}$, le code général des impôts, les lois de finances annuelles et toutes autres lois fiscales. D'une façon générale, la

1 Voir «Le scandale à 10 milliards de dollars », https://www.bbc.com/afrique/region-48501301 (Consulté le 3 juin 2019).

2 Le Fonds monétaire international (FMI) définit un pays comme étant « riche en ressources » lorsque les exportations de ressources naturelles non renouvelables comme le pétrole, les minéraux et les métaux représentent plus de $25 \%$ de la valeur des exportations totales du pays.

3 R. Auty, « Resource-Based Industrialization : Sowing the Oil in Eight Developing Countries », Clarendon Press, Oxford, 1990; G. Carbonnier, « Comment conjurer la malédiction des ressources naturelles? », Annuaire suisse de politique de développement, [En ligne], URL : http://journals.opened ition.org/aspd/123 (consulté le 19 juin 2019); G. Carbonnier, « La malédiction des ressources naturelles et ses antidotes », Revue internationale et stratégique, 2013/3 (n 91), p. 38-48. URL : https:// www.cairn.info/revue-internationale-et-strategique-2013-3-page-38.h, (consulté le 19 juin 2019).

4 Loi $n^{\circ}$ 96-00426/02/1996 portant code minier de la République togolaise, JO n ${ }^{\circ} 7$ du 26 février 1996, p. 1 et s. complété par Loi 03-012 14/10/2003 modifiant et complétant la loi n96-004 du 26 
collecte des taxes et droits ressortit aux prérogatives de l'office togolais des recettes (OTR). L'OTR a été créé par l'Assemblée nationale togolaise ${ }^{5}$. Il intègre les directions générales des Douanes et des Impôts au sein d'une structure unique. C'est un établissement public à caractère administratif, doté d'une autonomie de gestion administrative et financière. Il est chargé de recouvrer les impôts, taxes et droits de douanes pour le compte de l'État et des collectivités territoriales ${ }^{6}$.

La fiscalité se présente, pour l'État, comme une arme dans sa conception ${ }^{7}$. Cependant, elle se révèle en pratique comme une arme chargée à blanc. En effet, il est un secret de polichinelle que d'affirmer le défaut de clarté de la collecte des taxes et des droits des ressources naturelles. Des auteurs affirment à ce propos que : "le manque d'information concernant le partage de la rente minière entre les États et les investisseurs est un constat aisé en Afrique. Les bases de données existantes sont souvent insuffisantes pour mener une analyse détaillée des règles constituant les systèmes d'imposition africains appliqués aux secteurs des ressources naturelles, ce qui limite les travaux académiques et opérationnels $»^{8}$.

Les difficultés rencontrées dans la collecte de l'impôts sont sans doute liées à l'occultation des conventions qui lient les industries extractives et l'État. Ces difficultés mettent en lumière l'exigence d'un contrôle tiers sur la collecte des impôts et taxes. L'intérêt de ce contrôle se dédouble en ceci que l'effectivité d'un tel contrôle doit pouvoir être mesuré. Pour ce qui est de l'efficacité, elle doit permettre de vérifier la conformité du contrôle aux standards internationaux. Elle doit permettre, en outre, de voir s'il en ressort une réelle mâ̂trise de la fiscalité du secteur et s'il favorise la rentabilité pour l'économie nationale.

Le principe d'un tel contrôle n'est nullement discuté, dans la mesure où les textes législatifs et réglementaires ouvrent la voie à un tel contrôle. Néanmoins, l'on ne saurait faire l'économie de la double interrogation d'une part, sur l'effectivité d'un tel contrôle et, d'autre part, sur l'efficacité du contrôle. En substance, l'effectivité et l'efficacité du contrôle opéré sur la collecte des taxes et impôts tirées des ressources naturelles et minières sont-elles avérés?

février 1996 portant code minier de la république togolaise, $\mathrm{JO}^{\circ}{ }^{\circ} 33$ ter du 14 octobre 2003, p. 4 et s.NDLA : Le texte est actuellement en cours de révision.

5 Loi n ${ }^{\circ}$ 2012-16 du 14 décembre 2012 portant création de l'office togolais des recettes, JO du 15 décembre 2012, p. 2 et s.

6 Il a également en charge : 1) de conseiller ou représenter le gouvernement en matières fiscales et douanières; 2) promouvoir le consentement volontaire à l'impôt; 3) combattre la fraude, l'évasion fiscale et la corruption; 4) produire des statistiques sur les recettes collectées.

7 L'édition 2017 des statistiques des recettes publiques en Afrique réalisé par l'OCDE révèle une progression constante de la mobilisation des ressources domestiques dans les pays africains. Le rapport indique qu'en 2015, le ratio moyen des recettes fiscales rapportées au PIB pour les 16 pays couverts, dont le Togo, se fixait à $19.1 \%$, soit une hausse de 0.4 point par rapport à 2014 , http://oe.cd/re venue-statistics-in-africa-2017 (Consulté le 13 juin 2019).

8 B. Laporte, C. de Quatrebarbes, Y. Bouterige, « La fiscalité minière en Afrique : le secteur de l'or dans 14 pays de 1980 à 2015 », Revue d'économie du développement, 2015/4 Vol. 23, p. 1. 
La réponse à cette question n'a pas la force de l'évidence. L'on peut contrôler les taxes et les impôts tirés de l'exploitation des ressources naturelles et minières. En effet, le juge administratif et le juge judiciaire peuvent dans l'exercice de leur office contrôler la collecte des impôts et taxes tirés de l'exploitation des ressources naturelles. Au-delà de ce contrôle juridictionnel, l'on peut observer que le parlement, certains organes administratifs indépendants et même la société civile ont la possibilité de contrôler la collecte des impôts. Toutefois, la mesure de l'effectivité et l'efficacité de ces différents types de contrôle dans l'ordre juridique togolais semble être une gageure. Dès lors, l'analyse du contrôle de la fiscalité des ressources naturelles et minières du Togo peut être construite autour de deux grandes idées : un contrôle juridictionnel ineffectif (I) et non juridictionnel timoré (II).

\section{Un contrôle juridictionnel ineffectif}

Le contrôle juridictionnel s'entend du contrôle exercé par les juges sur les actes de l'administration. Ce contrôle peut se faire au plan administratif et au plan judiciaire. Dans les faits, l'on se rend compte que le contrôle des juridictions administratives est inexistant (A). Quant au contrôle judiciaire de l'activité fiscale, alors même qu'il est admis, il reste lettre morte (B).

\section{A. Le contrôle inexistant des juridictions administratives}

Le contrôle juridictionnel administratif se manifeste à travers l'intervention de certains organes : la cour des comptes et la chambre administrative de la cour suprême ${ }^{9}$. La Cour des comptes est la juridiction administrative ${ }^{10}$ par excellence de contrôle des deniers publics et du fisc. Elle est prévue par l'article 107 de la constitution togolaise et mise en œuvre par le décret 2009-049/PR du 24 mars 2009 portant application de la loi $\mathrm{n}^{\circ} 98-014$ du 10 juillet 1998 relative au fonctionnement de la cour des comptes. La loi $n^{\circ} 2019-003$ du 15 mai 2019 portant modification des dispositions des articles de la constitution du 14 octobre 1992 a prévu en son article 107 nouveau, des cours régionales de compte. Celles-ci sont appelées à jouer le rôle de cour des comptes dans leurs ressorts territoriaux.

A ce titre, la cour des comptes est chargée de certifier la régularité, la sincérité et la fidélité des comptes de l'État depuis la mise en œuvre de la loi organique relative aux lois de finances (LOLF) du $1^{\text {er }}$ août 2001. Elle est chargée de la même mission notamment pour les comptes des organismes nationaux du régime général de la sécurité sociale depuis la loi organique du 2 août 2005.

9 Dans l'organisation judiciaire togolaise, la chambre administrative de la Cour suprême joue le même rôle que le Conseil d'État en France ou au Sénégal.

10 Conformément à la directive $\mathrm{n}^{\circ}$ 06/2009/CM/UEMOA portant lois de finances au sein de l'UEMOA, les États membres sont dotés de Budgets Programmes. La Cour Comptes exerce désormais un contrôle sur la gestion des administrations en charge de l'exécution des programmes et dotations. 
La collecte de l'impôt et autres taxes relève de la compétence d'un établissement public administratif au Togo, l'OTR. Or, l'existence d'une juridiction administrative s'explique par la nécessité de juger et de contrôler l'administration afin de régler les conflits entre elle et les usagers. De manière générale, un administré peut s'adresser au juge administratif pour tout conflit l'opposant à une personne publique, notamment un établissement public administratif. Un tel recours permet au juge administratif de contrôler l'action de l'administration.

Les motifs pouvant conduire à une telle saisine sont assez variés. Il peut s'agir d'un recours contentieux en vue d'obtenir l'annulation totale ou partielle d'une décision administrative ${ }^{11}$, d'engager la responsabilité de la personne publique du fait d'un dommage subi par l'administré qui sollicite alors une indemnisation ${ }^{12}$; de contester la régularité des élections municipales, cantonales, régionales ${ }^{13}$ ou européennes. Les motifs qui nous intéressent en particulier sont ceux relatifs à un contentieux fiscal, à la contestation des impôts directs (impôt sur le revenu, impôt sur les sociétés, taxe d'habitation, taxe foncière...) et de la taxe sur la valeur ajoutée. Ils relèvent du juge administratif. La collecte des impôts et taxes fait donc l'objet d'un contrôle, par le biais de la saisine du juge administratif. Si sur le principe, l'existence d'un tel contrôle administratif n'est pas discutée, dans la pratique l'intervention du juge administratif est pour l'heure restée théorique. La justice administrative est balbutiante au Togo. Dans les faits, à l'heure actuelle aucune saisine de ce chef n'a été enregistrée par le juge administratif. L'effectivité d'un contrôle du juge administratif est donc aléatoire. Quid du juge judiciaire?

\section{B. L'incursion admise du juge judiciaire dans le contrôle fiscal}

Les activités de l'administration sont en principe jugées par les juridictions administratives compétentes ratione materiae. Cependant, pour des motifs variés et, dans un certain nombre de cas, le juge judiciaire peut revêtir les oripeaux du juge administratif ${ }^{14}$. De façon générale, on peut dire que le juge judiciaire est compétent pour les activités de l'administration ne présentant pas de caractère administratif (fonctionnement du service public de la justice, gestion privée des services publics ${ }^{15}$ ). Cependant, des exceptions à ce principe ont

11 Exemple : le refus d'une bourse de l'enseignement supérieur ou une mesure de police (ex : arrêté municipal interdisant la circulation dans une rue pour des raisons d'ordre public Cf. CCA de Nantes 29 juillet 2005, Association des commerçants citoyens de Dol de Bretagne.

12 Exemple : la responsabilité hospitalière à la suite d'une faute dans le traitement d'un patient (dommages liés à des travaux publics).

13 Au Togo, aux termes des articles 144 à 148 du code électoral, la chambre administrative de la Cour Suprême est seule compétente pour connaître du contentieux des élections locales.

14 Depuis le XIX ${ }^{\mathrm{e}}$ siècle et le développement de la gestion privée des services publics, le champ de compétences du juge administratif s'est restreint au profit du juge judiciaire.

15 Ce contrôle s'exerce d'abord au niveau des actes pris par une autorité administrative dans le cadre d'une situation juridique de droit privé. Tel est le cas des contrats de droit privé conclus par l'administration (ex : le contrat liant un médecin donnant des consultations libérales dans un hôpital 
étendu les attributions des tribunaux judiciaires, soit en vertu d'idées générales (état des personnes, liberté individuelle et propriété privée, responsabilité de l'administration), soit sans logique particulière (fiscalité indirecte, contraventions de voirie à l'exception toutefois de la taxe sur la valeur ajoutée qui relève du juge administratif) et en vertu d'une loi (décisions de l'Autorité des marchés financiers).

La justice judiciaire se trouve ainsi compétente pour connaître des contentieux relatifs à la fiscalité indirecte hormis ceux relatifs à la taxe sur la valeur ajoutée et aux contraventions de voirie. Cette possibilité est donc admise spécifiquement pour les droits de mutation à titre onéreux (DMTO), dus à raison de l'acquisition d'un immeuble par exemple. En matière de ressources minières et de ressources naturelles, l'hypothèse d'acquisition d'immeuble n'est nullement à exclure, dans la mesure où ceux-ci peuvent servir de support à l'exploitation de celles-là. La revue de la jurisprudence judiciaire togolaise a néanmoins permis d'établir qu'aucune action n'a été menée de ce chef.

\section{Le contrôle non juridictionnel timoré}

Le contrôle non juridictionnel consiste en un contrôle sur l'action administrative qui n'est exercé ni par le juge administratif ni par le juge judiciaire. Il peut être politique ou apolitique. Le contrôle politique apparaît inactif (A) alors que le contrôle apolitique est mi-figue mi-raisin (B).

\section{A. Un contrôle politique inactif}

Le contrôle politique est exercé par le parlement sur le pouvoir exécutif. Michel Ameller estimait que «c'est à l'étendue de leurs moyens de contrôle que se mesure aujourd'hui la force des assemblées parlementaires ${ }^{16}$, car « le contrôle parlementaire est l'un des piliers de l'État de droit. Le pouvoir exécutif et l'administration doivent demeurer sous le regard des citoyens pour éviter la dictature du guichet $»{ }^{17}$. Le Parlement contrôle l'action admi-

public à son patient est un contrat de droit privé). C'est également le cas des actes pris en matière de gestion du domaine privé des personnes publiques (ex : forêts domaniales).

La compétence du juge judiciaire s'exerce également sur certains services et particulièrement les services publics à caractère industriel et commercial, qui fonctionnent de manière semblable à des entreprises privées. Leur contentieux appartient au juge judiciaire (depuis l'arrêt Société commerciale de l'Ouest africain dit du Bac d'Eloka du Tribunal des conflits du 22 janvier 1921).

16 M. Ameller, Les questions, instrument du contrôle parlementaire, LGDJ, 1964, p. 9.

17 É. Thiers, « Le contrôle parlementaire et ses limites juridiques : un pouvoir presque sans entraves », Pouvoirs 2010/3 n 134, pages 71 à 81 https://www.cairn.info/revue-pouvoirs-2010-3-pag e-71.htm, (Consulté le 10 juin 2019). 
nistrative par des moyens d'information et d'investigation ${ }^{18}$. Les procédures d'information sont les questions écrites ${ }^{19}$, les questions orales ${ }^{20}$, les questions au gouvernement ${ }^{21}$.

Les pouvoirs d'investigations ne sont pas explicites dans la constitution togolaise. En revanche le règlement intérieur ${ }^{22}$ de l'Assemblée Nationale propose des dispositions pertinentes. Ainsi, selon l'article 35, l'Assemblée nationale peut constituer en son sein, des commissions spéciales pour un objet déterminé. De même, l'article 46 organise des missions d'information ou d'enquête. L'Assemblée nationale peut autoriser les commissions permanentes ou spéciales à désigner des missions d'information ou d'enquête sur les questions relevant de leur compétence. En 2016, le parlement du Burkina Faso ${ }^{23}$ a mené une enquête parlementaire sur la gestion des titres miniers et la responsabilité sociale des entreprises minières. Au Togo, aucune initiative de ce genre n'a pour l'heure été mise en œuvre. Dans la plupart des États africains tout comme en France, la concordance des majorités parlementaire et présidentielle fragilise ce contrôle parlementaire. Dans un tel contexte, il revient alors à l'opposition de jouer pleinement son rôle. Elle peut alerter l'opinion publique et même solliciter des actions judiciaires sur la question. Le rôle de l'opposition reste donc important pour impulser ce contrôle.

\section{B. Un contrôle apolitique mi-figue mi-raisin}

Le contrôle apolitique peut prendre plusieurs formes. Il concerne, entre autres, la hiérarchie interne, les Autorités administratives indépendantes, les organisations non gouvernementales, la société civile, les médias et les citoyens.

Relativement au contrôle interne, il s'agit d'un contrôle nécessaire pour veiller au respect du droit par l'administration et préserver ainsi l'État de droit. Le contrôle interne est un contrôle dont l'élément central est lié au pouvoir hiérarchique. Il se manifeste par le contrôle hiérarchique. Ainsi, tout supérieur hiérarchique peut modifier ou annuler les actes de ses subordonnés, lorsqu'ils ne sont pas conformes à la légalité ou lorsqu'ils ne lui paraissent pas opportuns.

Un contrôle peut être exercé par les autorités administratives indépendantes, par exemple : le Médiateur de la République. Ces autorités sont soustraites à tout pouvoir hiérarchique, ce qui justifie leur classement parmi les acteurs du contrôle externe. Au Togo

18 Par confort, les gouvernements cherchent naturellement à limiter la portée des contrôles parlementaires. Ils peuvent y parvenir en s'appuyant sur leur majorité qui s'autodiscipline et tient l'opposition à distance.

19 Article 96, al. 3 de la Constitution togolaise.

20 Idem.

21 Ibidem

22 Règlement intérieur de l'assemblée nationale togolaise, modifiée par la résolution du 22 janvier 2019.

23 La commission a été mise en place par la résolution nº19-2016/AN du 12 avril 2016, de mettre en place une commission d'enquête. 
c'est l'article 154 de la constitution qui institue un Médiateur de la République chargé de-de régler les conflits non juridictionnels entre les citoyens et l'administration. Une loi organique a organisé le statut et les attributions du Médiateur ${ }^{24}$. Dans l'exercice de cet office, il dispose d'un certain nombre d'outils qui lui permettent de contrôler par ricochet l'action administrative. En effet, conformément à l'article 7 de la loi organique ci-dessus citée, "le Médiateur de la République est chargé de recevoir, dans les conditions fixées par la loi les réclamations concernant le fonctionnement des administrations de l'État, des collectivités publiques territoriales, des établissements publics et de tout autre organisme investi d'une mission de service public dans leur relation avec les administrés ».

De même, "le Médiateur de la République ne peut intervenir dans une procédure engagée devant une juridiction, ni remettre en cause le bien-fondé d'une décision juridictionnelle. Cependant, il a la faculté de faire des recommandations à l'organisme mis en cause. Il peut en outre, en cas d'inexécution d'une décision de justice ayant acquis autorité de chose jugée, enjoindre à l'organisme mis en cause de s'y conformer dans un délai qu'il fixe ${ }^{25}$. Comme on peut l'observer à l'analyse des dispositions citées, le Médiateur dispose de moyens indirects lui permettant de contrôler l'action de l'administration fiscale voire de lui donner des injonctions. Cependant, à l'heure actuelle, l'étude du rôle du Médiateur de la République révèle qu'aucune saisine n'est à dénombrer relativement aux difficultés de collecte des impôts et taxes.

Le contrôle citoyen, quant à lui, est celui initié par les organisations non gouvernementales et la société civile. Dans le cas particulier des organisations non gouvernementales et relativement aux des ressources minières, il faut citer l'Initiative pour la Transparence dans les Industries Extractives (ITIE). Il s'agit d'une norme mondiale pour la promotion d'une gouvernance ouverte et redevable des ressources pétrolières, gazières et minérales.

La norme ITIE exige des États qu'ils publient des informations sur la chaîne de valeur extractive, de l'octroi des droits d'extraction aux revenus du gouvernement et à la façon dont ces revenus bénéficient à la population. L'ITIE cherche ainsi à renforcer la gouvernance publique et des entreprises, à promouvoir une bonne compréhension de la gestion des ressources naturelles et à fournir des données pour informer et mener des réformes permettant d'endiguer la corruption et aller vers une gestion plus responsable du secteur extractif $^{26}$. Au rang des exigences de la norme ITIE, Il faut citer les stipulations relatives à l'obligations de divulguer une description du cadre légal et du régime fiscal applicables aux in-

24 Loi organique $\mathrm{N}^{\circ} 2003-21$ du 9 décembre 2003 portant statut, attribution du médiateur de la république et composition, organisation et fonctionnement de ses services, $J O$ du 27 décembre 2003, p. 4 et s.

25 Article 8 de la loi organique précitée.

26 https://eiti.org/fr/qui-sommesnous (consulté le 14 février 2019). 
dustries extractives ${ }^{27}$, les taxes et revenus ${ }^{28}$. Grâce au rapports annuels publiés par l'ITIE, les informations relatives à la collecte des taxes et impôts liés aux industries extractives sont disponibles.

Les médias et la société civile sont également concernés par ce contrôle apolitique. Leur mobilisation par la sensibilisation et le lancement d'alertes pourrait contribuer à ce contrôle de la fiscalité des ressources minières et naturelles. Au total, la faiblesse actuelle du contrôle des divers acteurs précités ne favorise guère la transparence de la collecte des ressources fiscales.

27 L'article 2.1 intitulé Le cadre légal et le régime fiscal stipule : «a) Les pays mettant en ouvre l'ITIE doivent divulguer une description du cadre légal et du régime fiscal applicables aux industries extractives. Ces informations doivent comprendre une description succincte du régime fiscal, incluant le niveau de décentralisation fiscale, un aperçu des lois et de la réglementation pertinentes, et des informations sur les rôles et responsabilités des entités de l'État concernées.

b) Dans les domaines où le gouvernement entreprend des réformes, le Groupe multipartite est encouragé à s'assurer que celles-ci sont bien documentées », https://eiti.org/fr/document/exigence s-norme-itie-2016\#r2-1 (Consulté le 14 février 2019).

28 Article $4.1 \mathrm{https} / / /$ eiti.org/fr/document/exigences-norme-itie-2016\#r2-1 (Consulté le 14 février 2019). 